\title{
Gestión de recaudación tributaria municipal: Una visión cultural
}

\author{
Hector Manuel Suarez Rios \\ msuarez2004@hotmail.com \\ Gabriela del Pilar Palomino Alvarado \\ dpalominoal@ucvvirtual.edu.pe \\ Carlos Miguel Aguilar Saldaña \\ agua0122@hotmail.com \\ Escuela de posgrado \\ Universidad César Vallejo
}

\section{RESUMEN}

El estudio se caracteriza por conocer y analizar la gestión tributaria municipal que permita establecer un modelo de gestión efectiva para mejorar los ingresos tributarios. La justificación coincide con las afirmaciones de Palacio, O (2018) quien manifiesta que la gestión de los impuestos municipales y su relación con la cobranza de ingresos tiene una correlación muy significativa comparada a través de la buena gestión de los tributos. Investigación no experimental, descriptivo de corte transversal. Técnica aplicada encuesta e instrumento cuestionario, a colaboradores y contribuyentes, datos procesado y analizado mediante software SPSS vs 25. Como resultado se obtuvo que la recaudación y cultura tributaria están por debajo del $50 \%$, esto puede deberse a la falta de estrategias adecuadas en la gestión de los tributos municipales. Como conclusión se menciona que no se cuenta con un plan de cultura tributaria a los contribuyentes, ni existen canales adecuados para el pago de los tributos, sumándose a la falta de transparencia en dar a conocer a la población en que se invierten los impuestos pagados, originando que muchos contribuyentes no paguen oportunamente sus impuestos y se incremente la cartera de morosidad.

Palabras clave: Gestión; impuesto; tributo; recaudación tributaria. 


\title{
Management of municipal tax collection: A cultural vision
}

\begin{abstract}
The study is characterized by knowing and analyzing municipal tax management that allows establishing an effective management model to improve tax revenues. The justification coincides with the statements of Palacio, O (2018) who states that the management of municipal taxes and their relationship with the collection of income has a very significant correlation compared to the good management of taxes. Nonexperimental, descriptive cross-sectional research. Applied survey technique and questionnaire instrument, to collaborators and taxpayers, data processed and analyzed using SPSS vs. 25 software. As a result, it was obtained that the collection and tax culture are below $50 \%$, this may be due to the lack of adequate strategies in the management of municipal taxes. As a conclusion, it is mentioned that there is no tax culture plan for taxpayers, nor are there adequate channels for paying taxes, adding to the lack of transparency in informing the population in which the taxes paid are invested, causing that many taxpayers do not pay their taxes on time and the delinquency portfolio increases.
\end{abstract}

Keywords: Management; tax; tribute; tax collection.

Artículo recibido: 25 oct. 2020

Aceptado para publicación: 07 nov. 2020 Correspondencia: msuarez2004@hotmail.com.

Conflictos de Interés: Ninguna que declarar 


\section{INTRODUCCIÓN}

Los sistemas tributarios han sido y seguirán siendo en años venideros, objeto de profundas reformas y en el plano internacional se observa que el impuesto tributario no es considerado como un deber del ciudadano, por lo que no asumen a consciencia su importancia para el desarrollo de la sociedad (De Cesare, 2016), que, con una buena política transparente en la ejecución del gasto en actividades públicas, puede incrementar los ingresos municipales más de lo planificado.

En la región Latinoamericana, la recaudación por impuesto predial es inferior al $1 \%$ del producto bruto interno; siendo México, Guatemala y Ecuador los países de menor recaudación y los restantes con comportamiento no lineal; naciones como Costa Rica y otros de la región, han realizado inversiones considerables en el trabajo de revaluación de los inmuebles a través de la modernización del catastro nacional que vienen utilizando las municipalidades. Asimismo, en el Perú, la deficiente administración de tributos en los últimos diez años, ocasionó el establecimiento de sistemas de recaudación sólidos con módulos tributarios semiautomáticos en las grandes ciudades, que represento de alguna manera efectos satisfactorios (De Cesare, 2016). Situación que no se vio reflejada en las provincias o áreas rurales, cuyos resultados continúan siendo bajos.

A esto se suma, la evasión de los tributos que afecta significativamente al Estado y a la población del cual depende, originando que los gobiernos locales no cuenten con presupuesto suficiente para otorgar servicios básicos de calidad que la ciudadanía requiere, especialmente a los más vulnerables.

En el Perú, uno de los grandes problemas que se afronta, es el bajo nivel de recaudación y el incremento de la morosidad predial, situación que se agravó en este periodo de pandemia del COVID 19, al haberse declarado en estado de emergencia sanitaria por 90 días según D.S. No 008-2020-SA y las ampliaciones posteriores para mitigar el impacto social y económico negativo a la población. (Diario “E1 Peruano”, 2020).

Este contexto se observa de manera similar en la municipalidad provincial de Rioja, ya que no solo afronta un bajo nivel de recaudación tributaria, como se visualiza año a año, sino que también, ha tenido que enfrentar las normativas impuestas por el gobierno para prevenir la propagación del SARS-CoV-2, agudizando los ingresos en este rubro. La deficiente cultura tributaria de los contribuyentes, la espera de amnistías, es decir, 
eliminación o condonación de responsabilidad de pago; sumado a la carencia de estrategias o acciones de las autoridades y/o funcionarios para mejorar la cobranza, la poca transparencia en el manejo económico de la recaudación en los portales web, no reflejada en el desarrollo del distrito; son razones que impiden el cumplimiento de las metas fiscales y presupuestarias en la entidad.

En tal sentido, surge la necesidad de reorientar la gestión de recaudación tributaria municipal, para afrontar la situación crítica que vive actualmente, la región, el Perú y el mundo, promoviendo la cultura de pago responsable.

Ante esta problemática surge la siguiente interrogante ¿Cómo estará caracterizado la gestión de recaudación tributaria y la cultura de pago de los contribuyentes de la municipalidad provincial de Rioja?

El valor social del estudio, radica en la búsqueda de la equidad entre los ciudadanos riojanos, en satisfacer las necesidades de educación y salud en la provincia, así como, en la prestación de otros servicios, evitando que el contribuyente evasor goce de ventajas significativas frente al contribuyente cumplidor.

El objetivo es describir la gestión de recaudación tributaria y la cultura de pago de los contribuyentes de la municipalidad provincial de Rioja, resaltando las razones o nudos críticos que la dificultan.

\section{La recaudación tributaria municipal}

La variable se sustenta con las teorías aportadas por los diferentes autores, así tenemos a Rueda, J. quien definió que el deber tributario es el derecho público y vinculante entre las entidades públicas y los contribuyentes, según normativas de cumplimiento, siendo exigible coactivamente (2019). Asimismo, en el manual tributario del sector de economía, definió que cobranza tributaria forma la base principal de toda administración municipal, y radica en el cobro de los compromisos tributarios (2013).

Además, Justo, R., en su texto único ordenado (TUO) del código tributario del Perú, sostiene que la administración tributaria es la unidad encargada de gestionar la recaudación de los tributos municipales y son las únicas que administran las contribuciones y tasas municipales, excepto los impuestos que la ley les ordene (2019). $\mathrm{Y}$, el ente rector del sector economía, indica que las atribuciones de la administración 
tributaria local son registro y determinación de la deuda, cobranza tributaria, fiscalización y las sanciones según el código tributario aprobado por decreto supremo No 133-2013EF.

No obstante, Llontop (2017) manifestó que el procedimiento de recaudación consiste en la cobranza a consecuencia de los tributos liquidados de cada contribuyente según les pertenezca. Este procedimiento lo realiza la unidad organiza tributaria, quien tiene la responsabilidad de las cobranzas con adecuadas estrategias para efectivizar los mismos, ya que los bajos niveles de recaudación afectan al presupuesto institucional para la ejecución de los diferentes servicios públicos.

Al respecto, Rolando S. manifiesta que la administración tributaria municipal tiene la función de gestionar los recursos que generan la cobranza de impuestos, para lo cual debe conformar una cultura administrativa, cambiar de una gestión centralizada en procedimientos a una administración prestadora de servicios, poniendo interés en incrementar el pago voluntario de los contribuyentes (2019).

De igual forma, en la guía para el incremento de la recaudación tributaria del sector economía, expresa que la cobranza tributaria es facultad de toda administración tributaria, y radica en la admisión del dinero producto de la cancelación de las deudas tributarias. Asi mismo es facultada para que sea ejercida por terceros, como entidades del sistema bancario y financiero, previa celebración de contratos o convenios (2013).

No obstante, Cayo L. en su investigación manifestó que se requiere la implementación de diversas estrategias para alcanzar la eficiencia en la recaudación tributaria en cuanto a recursos financieros, humanos, materiales y tecnológicos para el respeto voluntario de los deberes tributarios (2017).

En la primera dimensión, referida a los impuestos municipales que según la ley de tributacion municipal D.L.776 aprobado por decreto supremo $\mathrm{N}^{\circ}$ 156.2004-EF en su artículo 5 indica, que los impuestos municipales son los tributos a favor de los gobiernos locales, cuyo cumplimiento no origina una contraprestación directa de la municipalidad al contribuyente y son el impuesto predial, impuesto de alcabala, impuesto al patrimonio vehicular, impuesto a las apuestas, impuesto a los juegos e impuesto a los espectáculos públicos no deportivos. Así mismo, en su artículo 8 de la mencionada ley, indica que la frecuencia del impuesto predial es por año y su valoración se determina por el valor total 
de los predios rústicos y urbanos del contribuyente, de responsabilidad de cada municipalidad (diario El Peruano, 2004).

Referente a la segunda dimensión, gestión de cuenta, el Ministerio de Economía y Finanzas (MEF) refiere a este proceso como el seguimiento de la deuda a fin de tener una idea clara y saber el progreso en la recuperación de los saldos por recaudar; el control de la deuda también nos indica los tiempos más adecuados para generar valores tributarios, y tomar acciones que ayuden a aumentar la cobranza impidiendo que la deuda prescriba (2016).

De igual manera en la guía metodológica del MEF, expresa que la gestión de la cuenta comprende las actuaciones de control y seguimiento de la deuda tributaria, independientemente de la etapa y estado de la cuenta. Abarca los procesos de cobranza ordinaria, cobranza coactiva y el control de la deuda para la emisión oportuna de los valores tributarios, el monitoreo adecuado de los fraccionamientos y el seguimiento de los saldos por cobrar (2019).

La gestión de la cuenta es medida por la cantidad de notificaciones preventivas de deuda proyectadas, de deudas realizadas, precoactivas de deuda proyectadas y realizadas, prescripciones tributarias proyectadas y realizadas; así como la proporción de morosidad proyectados y obtenida de los contribuyentes.

Según el Ministerio de Economía y Finanzas (2013) expresa que el seguimiento a la deuda es un proceso de gestión que requiere una labor ardua del personal para conocer cómo evoluciona el resultado de los pagos y planear trabajos a ejecutar en el futuro y corregir aquello que no esté brindando resultados. Referente a las notificaciones preventivas, éste se realiza dando a conocer al contribuyente su deuda desde que se determina hasta el vencimiento del plazo indicado. Asimismo, las notificaciones precoactivas se originan cuando no se paga oportunamente una deuda, por lo tanto, debiendo informar al contribuyente sobre su obligación y las implicancias que conlleva su no ejecución (intereses, gastos de cobranza y otros), que afectan al deudor. De igual forma, Sandoval, R. (2019) dio a conocer que la prescripción no es un mecanismo de término de la deuda tributaria, sino un medio de defensa que posibilita al deudor tributario se libere de una determinada obligación en virtud del transcurso del tiempo, siendo su fundamento la seguridad jurídica. Finalmente, la morosidad según Diaz, L. (2014), indica que cuantifica 
la demora en los pagos, considerado ratio de interés en la rama de las finanzas. Por tal motivo se puede considerar deudor a todo ciudadano o empresa que no ha cumplido con su obligación en un periodo de tiempo y deja de serlo cuando realice la cancelación.

Por tanto, en el código tributario aprobado por decreto supremo 133-2013-EF hace referencia a labores de la administración tributaria, establecida como la obligación, acciones para realizar la cancelación y aplicación de sanciones antes que prescriba a los cuatro años, y a los seis años para quienes no hayan presentado la declaración respectiva (Diario El Peruano, 2013).

Referente a cobranza coactiva, según Cabanellas, (1989) conceptualizó que es competencia de la administración tributaria recuperar las deudas tributarias, haciendo uso de normativas, que puede ser con incautación de bienes muebles o inmuebles, propiedad de los deudores con el objetivo de recuperar deudas.

En cuanto, a la tercera dimensión relacionado al registro de nuevos contribuyentes, en la guía del MEF (2019), señala que es un macroproceso que permite mantener un correcto registro de la información utilizada en administración tributaria para el cálculo de la base imponible y del monto del impuesto predial de cada contribuyente. Además, señala que su registro y determinación, son hechos que se encuentran gravados según normativa, identificando al deudor a pagar un tributo, determinando para ello la base imponible y el importe a pagar. Esta función es propia de la administración tributaria, estando prohibido ceder estas funciones a terceros. Asi mismo, se puede determinar la deuda predial con la presentación de las declaraciones juradas por parte del contribuyente.

Asi mismo, en el decreto legislativo 776 - Ley de Tributación Municipal, aprobado por Decreto Supremo No156-2004-EF indica que la base imponible para la determinación del impuesto predial está formada por el valor total de las propiedades del contribuyente situados en cada jurisdicción distrital.

En relación a la cuarta dimensión, meta predial, es un instrumento de incentivos presupuestales direccionado al presupuesto por resultado (PPR), dirigido por el sector economía que impulsa las mejoras para una calidad en servicios públicos en los gobiernos locales de todo el país. El plan de incentivos transmite asignaciones presupuestales a las entidades públicas por cumplir metas en un lapso de tiempo determinado, metas 
formuladas por instituciones públicas del estado, siendo la finalidad obtener los resultados esperados.

Sin embargo, en la guía del programa de incentivos al perfeccionamiento de la gestión municipal del sector economía - MEF (2019), indica que el plan de incentivos se desarrolló en tres procesos: diseño de metas, asistencia técnica para el cumplimiento de metas y la evaluación de su cumplimiento. Cada proceso tiene un documento normativo donde se detalla las metas, el otorgamiento de recursos, las guías de las metas, los resultados de cumplimiento y la transferencia de recursos. La preparación de toda la documentación conlleva a un trabajo constante con los sectores que tienen la responsabilidad del cumplimiento de metas, de acuerdo a la asistencia técnica del MEF en cuanto al diseño de las mismas y documentos relacionados. La meta predial es medida por el porcentaje proyectado de la meta predial del plan de incentivos y el total de porcentaje recaudado.

La quinta dimensión es la gestión recaudación tributaria, el cual permite conocer desde la perspectiva de los colaboradores como se realizó las acciones que permitieron lograr la recaudación. Esta dimensión es medida por la ejecución de acciones para promocionar la cultura tributaria, se da a conocer a la población en que se utilizan los pagos de los impuestos que realizan los contribuyentes, los contribuyentes realizan voluntariamente su declaración predial anual, se brinda algún estímulo o reconocimiento por el pago puntual, existen canales de pago en línea con instituciones financieras para impuestos, se brinda cursos de capacitación al personal de rentas, se asignan recursos para las funciones de recaudación tributaria, se realizan los catastros urbanos y rurales periódicamente, se realizan fiscalizaciones tributarias a los predios y si se difunde en el portal institucional orientación sobre tramite de solicitudes tributarios.

\section{Cultura tributaria}

Según la Superintendencia Nacional de Administración Tributaria (SUNAT), indica, que en toda Latinoamérica y particularmente en el Perú, está consignada que todos los ciudadanos comprendan y asuman que la tributación es una responsabilidad cultural. Que se debe formar desde la etapa escolar, desarrollando hábitos, actitudes, conductas, valores, entre otros, que serán determinantes para su actuar como ciudadano y contribuyente. Existe aún desconfianza en la gestión adecuada de los recursos del estado, 
lo que influye en el desempeño de las obligaciones del contribuyente. Por ello, es importante que desde la etapa escolar se le enseñe al niño a comprender porque debemos exigir nuestro comprobante de pago, qué pasa con el dinero que se recauda, donde podemos ver el uso de dicho dinero y que podemos hacer para lograr que los demás puedan sumarse a éstas conductas.

En su tesis doctoral de Lujan R. (2019) indica que la cultura es el resultado de muchos años de historia, producto de la contribución de todos los pueblos. En consecuencia, la cultura hace referencia a los valores que interactúan las personas, de acuerdo a las normas que establecen.

La cultura tributaria debe ser medida a través de: a) sensibilización y educación sobre tributación, b) aceptación del pago del impuesto predial, c) conocimiento sobre el uso de los pagos del impuesto predial, d) cancelación de impuestos sujetos a la ley u ordenanzas municipales, e) importancia de la cancelación del impuesto predial, f) responsabilidad en declaración anual del predio, g) reconocimiento o estimulo por el pago oportuno del impuesto y h) ordenanzas municipales con beneficios tributarios.

De igual manera, en el TUO en su artículo 55 indica que la facultad de recaudación de tributos es función de la administración tributaria recaudar los tributos. A tal efecto, podrá pactar directamente los servicios de las entidades del sistema bancario y financiero, asi como de otras entidades para recibir el pago de deudas a tributos administrados por aquella. Los convenios podrán incluir la autorización para recibir y procesar declaraciones y otras comunicaciones dirigidas a la administración.

\section{ESTRATEGIAS METODOLÓGICAS}

Investigación no experimental, descriptivo, prospectivo de corte transversal, porque se recogió información y se estudió la incidencia de la situación actual para contribuir en implementación de estrategias efectivas de mejora en la recaudación tributaria. La población estaba conformada por el funcionario de la oficina de rentas, colaboradores de rentas y contribuyentes. La muestra estuvo conformada por 1 funcionario de rentas, 6 colaboradores de rentas y 118 contribuyentes de la ciudad de Rioja.

Se utilizó el cuestionario sobre aspectos de la variable recaudación tributaria en las dimensiones de impuestos, gestión de cuenta, registro de nuevos contribuyentes, meta predial y gestión recaudación tributaria. 


\section{RESULTADOS}

\section{Recaudación Tributaria}

La cantidad ejecutada anual de recaudación por impuesto a alcabala representa el $81.8 \%$ de lo proyectado $(\mathrm{RP}=220,000.00, \mathrm{RE}=180,000.00)$, obteniendo una brecha del $18.2 \%$ en el año 2019. De igual manera la cantidad ejecutada anual por impuesto al patrimonio vehicular representa un $78.1 \%$ de lo proyectado $(\mathrm{RP}=32,000.00, \mathrm{RE}=25,000.00)$, obteniéndose una brecha del $21.9 \%$ y finalmente la cantidad ejecutada anual por impuesto predial representa un $60.0 \%$ de lo proyectado $\left(\mathrm{RP}=1^{\prime} 200,000.00, \mathrm{RE}=720,000.00\right)$, obteniéndose una brecha del $40 \%$.

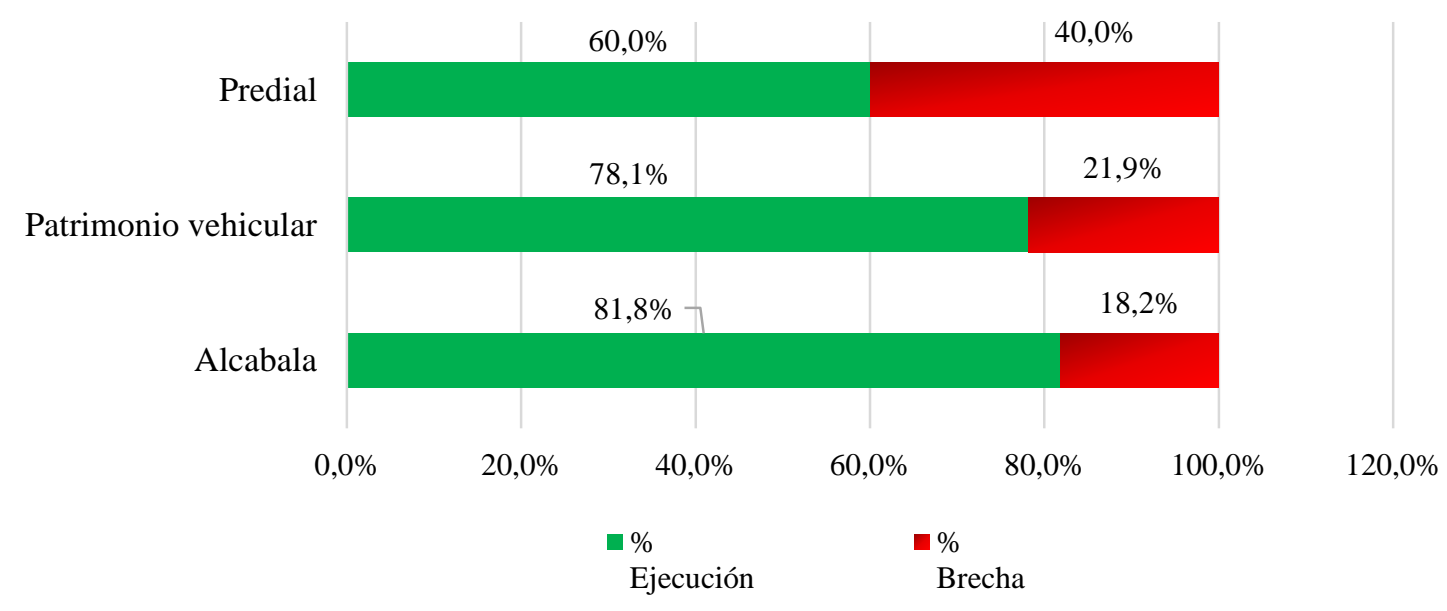

Figura 1. Ejecución y brechas de la recaudación tributaria

Fuente: Cuestionario aplicado al funcionario de rentas de la municipalidad provincial de Rioja.

\section{Cuentas por cobrar}

La cantidad anual ejecutada de prescripciones tributarias, representa el $91.7 \%$ de lo proyectado $(\mathrm{CP}=240, \mathrm{CE}=220)$, obteniendo una brecha del $8.3 \%$ en el año 2019. De manera similar la cantidad anual ejecutada de notificaciones precoactivas, representa el $88.6 \%$ de lo proyectado $(\mathrm{CP}=350, \mathrm{CE}=310)$, siendo la brecha del $11.4 \%$, igualmente la cantidad anual ejecutada de notificaciones preventivas representa el $80.0 \%$ de lo proyectado $(\mathrm{CP}=2,500, \mathrm{CE}=2,000)$, con una brecha del $20 \%$ y finalmente el porcentaje de morosidad de los contribuyentes proyectada fue del $35 \%$, incrementándose a un $40 \%$ en el año de estudio. 


\section{Gestión de la recaudación tributaria de los colaboradores}

De los 6 colaboradores encuestados, el 100\% (6) refiere que la municipalidad no brinda ningún estímulo o reconocimiento por el pago puntual de los contribuyentes, ni existen canales de pago en línea o con instituciones financieras por concepto de impuestos, tampoco se brinda cursos de capacitación al personal de rentas sobre tributación municipal, no se realizan los catastros urbanos y rurales periódicamente, ni se difunde en el portal web institucional sobre orientación de formularios y trámites de solicitudes de asuntos tributarios, respectivamente.

Asimismo, el 83,3\% (5), considera que "Nunca o casi nunca" los contribuyentes realizan voluntariamente su declaración predial anual, esto podría deberse, a que la municipalidad no da a conocer a la población en qué se utilizan los pagos de los impuestos que realizan los contribuyentes.

Respecto a la asignación de recursos necesarios y oportunos para las funciones de recaudación tributaria, el 50,0\% de los colaboradores refiere que "Nunca o casi nunca" se realiza esta acción, con tendencia a que se realiza "Algunas veces" en la misma proporción.

En cuanto a las acciones ejecutadas por la municipalidad para promocionar la cultura tributaria, el 50,0\% (3) observa que "A veces" se realiza, con tendencia a "Nunca y casi nunca" en un 33,3\% (2) y finalmente en relación a las fiscalizaciones tributarias de los predios, el 50.0\% (3) refiere que "A veces" se realizan, con tendencia a "Nunca y casi nunca" en un $33,3 \%(2)$. 


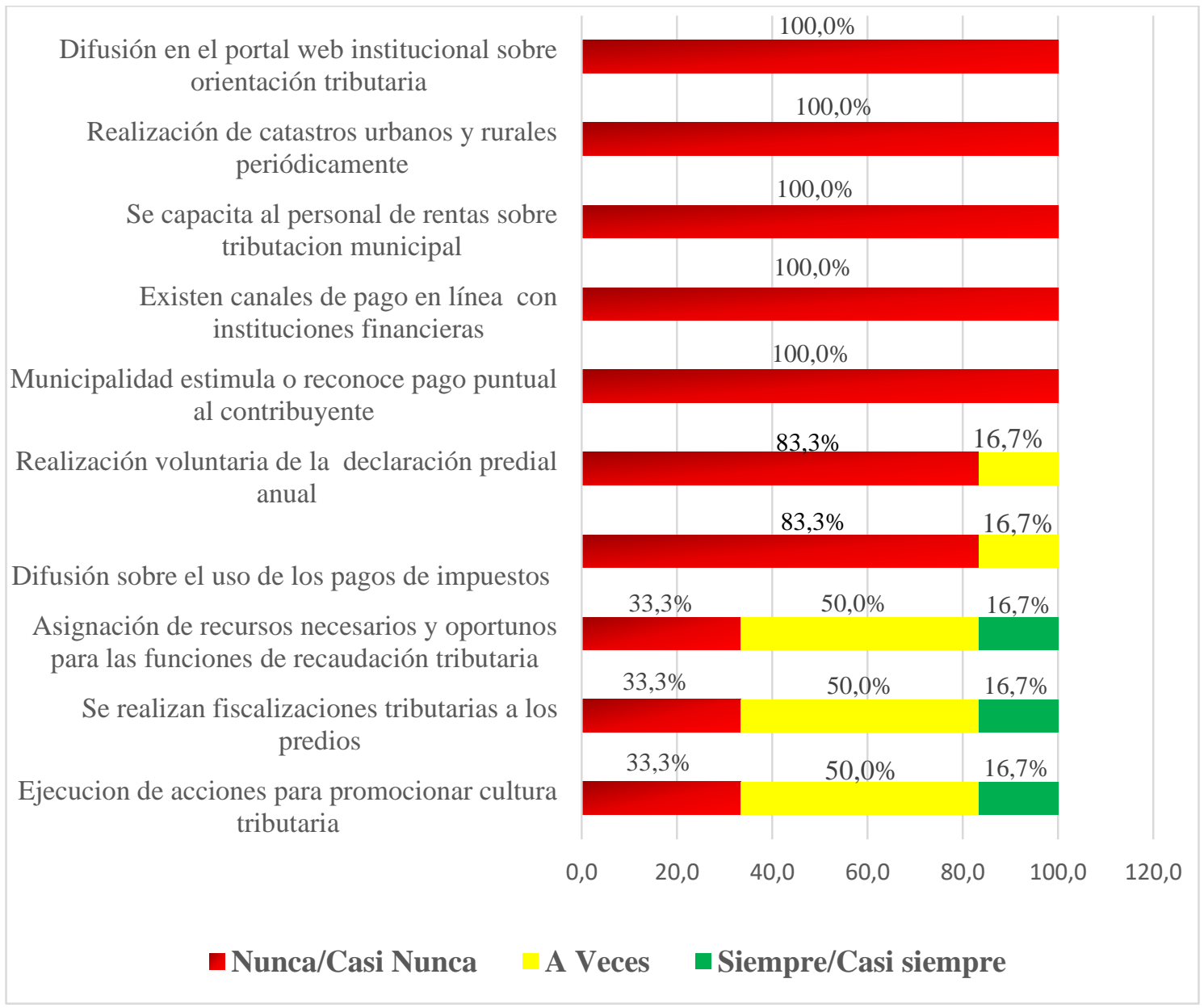

Figura 2. Gestión de la recaudación tributaria

Fuente: Cuestionario aplicado a colaboradores de la municipalidad provincial de Rioja.

\section{Caracterización de la cultura tributaria de los contribuyentes}

De los 118 contribuyentes encuestados, el 92.4\% (109) refiere que están "De acuerdo o muy de acuerdo" que la población debe conocer, en que se utilizan los pagos por impuesto predial que realizan los contribuyentes, el 91.5\% (108) manifestaron que consideran muy importante que se realicen la cancelación el impuesto predial, ya que el incumplimiento de pago de impuestos, generará retraso en el desarrollo del distrito, para ello el $89.8 \%$ (106) manifestaron que la municipalidad debe brindar reconocimiento o estímulo por el pago oportuno de impuesto municipales, asi mismo brindar beneficios para la regularización de deudas tributarias.

De igual manera, $83.9 \%$ (99) refirieron que se debería cancelar el impuesto predial en los plazos establecidos por ley u ordenanza municipal, siendo el $81.4 \%$ (96) que requieren 
ser sensibilizados, educados y que se promocione la cultura tributaria a la población, con la finalidad de que el contribuyente realice su declaración anual de las características de sus predios $(69.5 \%, 82)$.

Finalmente, el 58.5\% (69) indicaron que mayormente se debe de pagar los impuestos cuando existen beneficios tributarios, debido a que solo el 49.2\% (58) manifestaron tener las condiciones económicas para asumir el pago de impuesto predial y el 42.4\% (50) indican que no aceptan el importe de pago por impuesto predial emitido por la oficina de rentas.

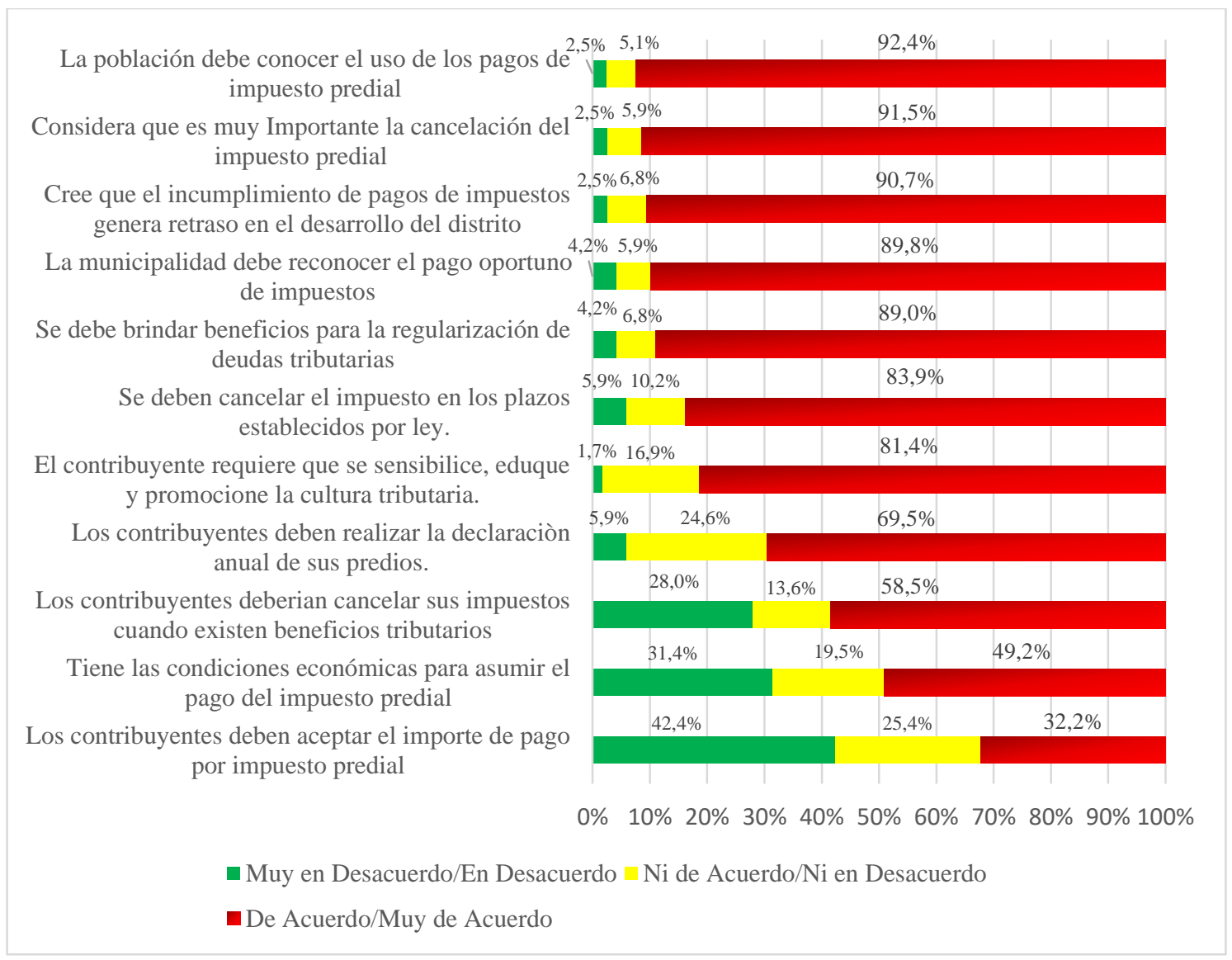

\section{Figura 3. Cultura tributaria de los contribuyentes}

Fuente: Cuestionario aplicado a contribuyentes de la ciudad de Rioja.

\section{Indicadores de gestión del servicio tributario}

Del total de contribuyentes encuestados (118), el 90.7\% (107) refiere que están "De acuerdo o muy de acuerdo" que la municipalidad debe de comunicar oportunamente por medios publicitarios a los contribuyentes sobre los vencimientos tributarios, de igual manera el 84.7\% (100) manifestaron que se debe de implementarse canales de atención 
suficientes para el pago de los tributos, implementando pagos en línea de impuestos con diferentes entidades financieras $(83.9 \%, 99)$.

De forma similar, el 75.9\% (109) refiere que se debe ejecutar acciones apropiadas para cobranza de impuestos, y finalmente el 75.4\% (89) manifestó que la municipalidad debe de contar con personal capacitado para la recaudación y orientación al contribuyente.

\section{DISCUSION}

Considerando que la presente investigación tiene como objetivo conocer y analizar la gestión tributaria municipal; y luego de realizar el análisis de resultados, se observa que se requiere de manera prioritaria un modelo de gestión efectiva, debido a que la forma que se vienen gestionando la recaudación, no son las adecuadas y existen necesidades que los contribuyente requieren, conllevando a obtener alto porcentaje de morosidad de recaudación, y con ello existe el riesgo de no poder cumplir en futuro las metas mínimas de recaudación que fija el Ministerio de Economía y Finanzas, como programa de incentivos municipales y que además no se tenga los recursos directamente recaudados necesarios para la adquisición de bienes y servicios.

Las oficinas de administración tributaria, tienen una gran responsabilidad, debido a que son las responsable de determinar los impuestos de cada uno de los predios, originando deudas por cobrar en beneficio a la entidad municipal, por tal motivo se debe conocer a menudo las normas internas de las funciones de cada colaborador como también las normativas externas actualizadas que brinda el ministerio de economía y finanzas sobre tributacion municipal, originando que se debe contar con personal especializado en esta rama.

Por su parte, Vera, P. (2019) en su trabajo de investigación llega a la conclusión que la implementación del sistema fiscalización tributaria, ha permitido que mejore la cobranza de ingresos de manera sustancial.

En el primer objetivo específico: Caracterizar la gestión de la recaudación tributaria de la municipalidad provincial de Rioja; se aplicó tres cuestionarios que estuvo dirigido al funcionario de rentas (1), a los colaboradores (6) y a los contribuyentes (118), considerando para la prueba de confiabilidad a 10 encuestados, siendo procesados a través 
del programa estadístico SPSS vs 25, el cual se obtuvo en la variable de recaudación tributaria 0.808 de confiabilidad aplicando el alfa de Cronbach.

Al realizar el análisis de estudio, se obtuvo que existen nudos críticos relevantes que limitan la efectiva gestión de recaudación tributaria, siendo estos: ejecución del $60 \%$ de la recaudación proyectada por impuesto predial, quedando una brecha por mejorar del $40 \%$, debido tal vez, a que los usuarios no están debidamente comunicados porque no se difunden en el portal web información sobre orientación y trámites de solicitudes de asuntos tributarios, ni se realizan los catastros urbanos y rurales periódicamente, tampoco se brindan cursos de capacitación al personal sobre tributación municipal, tampoco existen canales de pago en línea con instituciones financieras para pago de sus predios; asi mismo, no se brinda estímulos o reconocimiento por el pago puntual a los contribuyentes, ni tampoco ellos realizan la declaración anual predial voluntariamente, pudiendo considerarse a que la municipalidad no da a conocer a la población en que se utilizan los pagos de impuestos, lo que genera retraso en el desarrollo del distrito.

A esto se suma, que la entidad, no comunica oportunamente por medios publicitarios los vencimientos tributarios, ni tampoco brinda estímulos o reconocimiento por el pago puntual o beneficios para la regularización de las deudas tributarias, situación que podría mejorar si se implementa canales de atención suficientes para el pago de impuestos en línea o con instituciones financieras, aunado a la sensibilización, educación y promoción de la cultura tributaria, así como, personal capacitado para la implementación de acciones adecuadas en la cobranza de impuestos.

De los resultados obtenidos, se puede comparar con la investigación de Ibeth, G. (2018) donde refiere que la recaudación tributaria está por debajo de las metas, esto a causa de los beneficios tributarios tales como las amnistías y la falta de cultura tributaria de los contribuyentes y las fiscalizaciones prediales inciden negativamente en la recaudación de los tributos. De forma similar, Palacio, O (2018), estipula en su investigación que existe una correlación muy significativa comparada a través de la buena gestión de los tributos con la cobranza de ingresos municipales.

Es necesario precisar, una oficina tributaria bien gestionada ayuda a tener una eficiente captación de recursos financieros para cumplimento de los objetivos, de esa manera destinarlo responsablemente al desarrollo de la ciudad en beneficio de la población 
Riojana. Asimismo, los resultados, nos conduce a la imperiosa necesidad de implementar un adecuado modelo de gestión efectiva basado en desarrollar cultura tributaria en la población para mejorar los ingresos financieros de la municipalidad; y por ende del servicio y desarrollo hacia la población.

\section{CONCLUSIÓN}

De los resultados obtenidos de la caracterización de la variable recaudación tributaria, se pretende promover la formación de cultura de pago responsable que conlleve a incrementar los ingresos en bien del desarrollo de la ciudad.

La recaudación tributaria tiene una brecha por cobrar de un $40 \%$ de morosidad; esto puede deberse a la falta de mecanismos o acciones adecuadas que no se tiene implementado en la municipalidad y hace que los contribuyentes no realicen los pagos oportunamente.

La gestión de la recaudación tributaria presenta algunas deficiencias, como no se cuenta un plan de cultura tributaria a los contribuyentes y muchos de ellos indican que no se les brinda facilidades ni existen canales de pago adecuados de los impuestos; esto se suma a la falta de comunicación o promoción de la municipalidad hacia los contribuyente, originando una baja transparencia tributaria en dar a conocer a la población en que se invierten los impuestos pagados por los mismos contribuyentes.

\section{LISTA DE REFERENCIAS}

Amasifuén, R. (2015) Importancia de la cultura tributaria en el Perú. Recuperado de https://bit.ly/2Mb25tN

Cabezas, E., Andrade, D. y Torres, J. (2018) Introducción a la metodología de la investigación científica. (Libro). ISBN: 978-9942-765-44-4, Universidad de las Fuerzas Armadas - Ecuador.

Caetano, C. y Ávila, M. (2017) Relation between the government transfers, own tax collection and education index of the municipalities of the state of Minas Gerais. (Scientific Article). Universidade Federal de Uberlândia, Brazil. DOI: 10.1590/0034-7612174433. Recovered from: https://www.scopus.com/results/results.uri?cc=10\&sort=plf$\mathrm{f} \& \mathrm{src}=\mathrm{s} \& \mathrm{st} 1=$ Recaudaci\%c3\%b3n+Tributaria + Municipal $\&$ nlo= $=\& n l r=\& n l s=\& s i$

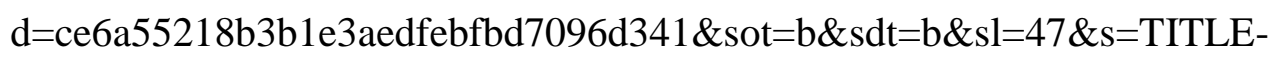


ABS-KEY\%28Recaudaci\%c3\%b3n+Tributaria+Municipal\%29\&ss=plf-f\&ps=rf\&editSaveSearch=\&origin=resultslist\&zone=resultslist

Claudia, C. (2016) Sistemas del impuesto predial en América Latina y el Caribe. Hollis. United States of América. Lincoln Institute of Land Policy.

DECRETO SUPREMO No 367-2017-EF. (2017). El Peruano. Aprueban los procedimientos para el cumplimiento de metas y la asignación de los recursos del Programa de Incentivos a la Mejora de la Gestión Municipal del Año 2018. Lima, Perú.

Espinoza, S. y Martínez, J. y Martell, C. (2018) Why some municipalities in Mexico are better property tax collectors than others?. (Scientific Article). Centro de Investigación y Docencia Económicas A.C. ISSN: 14051079. Recovered from: https://www.scopus.com/record/display.uri?eid=2-s2.0$85059462708 \&$ origin $=$ resultslist $\&$ sort $=$ plf$\mathrm{f} \& \mathrm{src}=\mathrm{s} \& \mathrm{st} 1=+$ municipal + tax + collection $+\mathrm{in}+$ latin+america\&st $2=\& \operatorname{sid}=7 \mathrm{cbc} 9 \mathrm{be}$ c885c15c2f6487ae5cae32eef\&sot=b\&sdt=b\&sl=57\&s=TITLE-ABS-

KEY\%28+municipal+tax+collection+in+latin+america\%29\&relpos=0\&citeCnt $=0 \&$ searchTerm $=$

Florián Gómez, S. E. (2017). Cultura tributaria y calidad de la gestión recaudatoria en la gerencia de rentas de la Municipalidad Provincial de Barranca 2016 Lima - Perú.

Gomez, S. E. F. (2017). Cultura tributaria y calidad de la gestión recaudatoria en la gerencia de rentas de la Municipalidad Provincial de Barranca, 2016. Recuperado de: http://repositorio.ucv.edu.pe/bitstream/handle/UCV/6188/Florián_GSE.pdf? sequence $=1$

Gutiérrez, MU (2018) The limits of property tax collection in the urban municipalities of Mexico: A case study. DOI: 10.24201/edu.v33i3.1741. Colegio de Mexico, A.C. Recovered from: https://www.scopus.com/record/display.uri?eid=2-s2.0$85051712913 \&$ origin $=$ resultslist\&sort $=$ plf -

$\mathrm{f} \& \mathrm{src}=\mathrm{s} \& \mathrm{st} 1=$ municipal + tax + collection $\& \mathrm{st} 2=\& \operatorname{sid}=7 \mathrm{cbc} 9 \mathrm{bec} 885 \mathrm{c} 15 \mathrm{c} 2 \mathrm{f} 6487 \mathrm{ae} 5$ cae32eef $\&$ sot $=b \& s d t=b \& s l=39 \& s=$ TITLE-ABS -

KEY\%28municipal+tax+collection\%29\&relpos=16\&citeCnt=0\&searchTerm= 
Hernández, R., Fernández, C. y Baptista, M. (2014) "Metodología de la investigación”. (Libro). Sexta Edición, ISBN: 978-1-45. McGraw-Hill/Interamericana Editores S.A. - México.

Huamán, H. (2019). La cultura tributaria y la recaudación del impuesto predial en la Municipalidad Provincial de Casma, 2019. (Tesis). Recuperado de: http://repositorio.ucv.edu.pe/handle/20.500.12692/41306

Hummel, Lurita y Reynoso (2016). "La morosidad y la recaudación del impuesto predial en la Municipalidad Distrital de San Miguel periodo 2011 - 2015”. Lima, Perú.

Iregui, A., Melo, L. y Ramos, J. (2005) El impuesto predial en Colombia: factores explicativos del recaudo. (Revista). ISSN: 0123-5362, Universidad del Rosario Bogotá - Colombia. Recuperado de: https://www.redalyc.org/pdf/5095/509555103002.pdf

Lizana, K (2017). La cultura tributaria y su impacto en el cumplimiento de las obligaciones tributarias de rentas personales de la población de la región Lima, 2017. (Tesis de Licenciatura). Universidad San Martín de Porres. Perú Recuperado de: http://www.repositorioacademico.usmp.edu.pe/bitstream/usmp/3099/1/lizana_rk 1.pdf

Lujan, R. (2019). La cultura tributaria y el comportamiento de los delitos tributarios en el Perú: periodo 2012-2016. Escuela de Posgrado de la Universidad Nacional de Trujillo. La Libertad.

Ly, T. Paty, S. (2020). Local taxation and tax base mobility: Evidence from France. (Scientific Article). DOI: 10.1016/j.regsciurbeco.2019.01.010. Recovered from https://www.scopus.com/record/display.uri?eid=2-s2.0-

85062185117\&origin=resultslist\&sort=plf-

$\mathrm{f} \& \mathrm{src}=\mathrm{s} \& \mathrm{st} 1=$ property+taxation $\& \mathrm{st} 2=\& \mathrm{sid}=288 \mathrm{~d} 7 \mathrm{e} 35462 \mathrm{ba0d} 2993926702 \mathrm{f} 357$

$113 \&$ sot $=\mathrm{b} \& \mathrm{sdt}=\mathrm{b} \& \mathrm{sl}=32 \& \mathrm{~s}=\mathrm{TITLE}-\mathrm{ABS}-$

KEY\%28property+taxation\%29\&relpos=17\&citeCnt=0\&searchTerm $=$

Manganelli, B. y Morano, P. (2020). The effect of taxation on investment demand in the real estate market: The Italian experience. (Scientific Article). DOI: 10.3390/BUILDINGS10070115. Recovered from https://www.scopus.com/record/display.uri?eid=2-s2.0- 
$85088451637 \&$ origin $=$ resultslist\&sort=plf-

$\mathrm{f} \& \mathrm{src}=\mathrm{s} \& \mathrm{st} 1=$ property+taxation $\& \mathrm{st} 2=\& \mathrm{sid}=288 \mathrm{~d} 7 \mathrm{e} 35462 \mathrm{ba0d} 2993926702 \mathrm{f} 357$

$113 \&$ sot $=\mathrm{b} \& \mathrm{sdt}=\mathrm{b} \& \mathrm{sl}=32 \& \mathrm{~s}=$ TITLE-ABS -

KEY\%28property+taxation $\% 29 \&$ relpos $=7 \&$ citeCnt=0\&searchTerm $=$

Maurélio, S. y Oliveira, E. (2011) A repartição tributária dos recursos do ICMS nos municípios da Região Metropolitana de Curitiba. (artigos). ISSN: 0034-7612. Recuperado de: https://www.scielo.br/scielo.php?script=sci_arttext\&pid=S0034$76122011000200008 \& \operatorname{lng}=\mathrm{en} \& n r m=\mathrm{iso}$

MEF (2004). “Texto Único Ordenado de la Ley de Tributación Municipal”. D.S.1562004-EF. Normas legales - Diario el Peruano - Perú.

MEF (2013). “Texto Único Ordenado del Código Tributario”. D.S.133-2013-EF. Normas legales - Diario el Peruano - Perú.

MEF (2018). “Guía para el registro y determinación del impuesto predial”. MEF. Lima, Perú.

MEF (2018). "Manuales para la mejora de la recaudación del impuesto predial. Manual № 4: Cobranza". 2da edición. Lima, Perú.

MEF (2018). "Manuales para la mejora de la recaudación del impuesto predial. Manual № 5: Declaración Jurada". 2da edición. Lima, Perú.

Ministerio de Economía y Finanzas. (2019). Programa de incentivos. Recuperado de internet, https://www.mef.gob.pe/es/incentivos-para-gobiernos-locales-yregionales/programas-de-incentivos-municipales-a-la-mejora-de-la-gestionmunicipal.

Oliver, N. (2016) La recaudación tributaria y su impacto en el desarrollo social de la región La Libertad periodo 2000-2013. Universidad Nacional de Trujillo.

Quispe, A. V. F. (2018). La cultura tributaria y su incidencia en el pago del impuesto predial del Asentamiento Humano 7 de Octubre de la Municipalidad del distrito de El Agustino 2017. Universidad Cesar Vallejo.

Sánchez Talanquer, M. (2020) One-Eyed State: The Politics of Legibility and Property Taxation. (Scientific Article). Cambridge University, United Stated. DOI: 10.1017/lap.2020.7. Recovered from https://www.scopus.com/record/display.uri?eid=2-s2.0$85087958537 \&$ origin=resultslist\&sort=plf- 
$\mathrm{f} \& \mathrm{src}=\mathrm{s} \& \mathrm{st} 1=$ property+taxation $\& \mathrm{st} 2=\& \mathrm{sid}=288 \mathrm{~d} 7 \mathrm{e} 35462 \mathrm{ba0d} 2993926702 \mathrm{f} 357$ $113 \&$ sot $=b \& s d t=b \& s l=32 \& s=$ TITLE-ABS -

KEY\%28property+taxation $\% 29 \&$ relpos $=3 \&$ citeCnt=0\&searchTerm $=$

Serrano. (2017). Análisis de la cultura tributaria y su influencia en el cumplimiento de las obligaciones tributarias de los arrendadores de rentas de primera categoría arrendamiento de predios de personas naturales en la Provincia de Abancay 2016. Escuela de Posgrado de la Universidad Nacional del Altiplano. Puno.

Thesari, SS. y Troyano, F. y Batistus, DR. (2019) A decision model for municipal resources management. (Scientific Article). DOI: 10.1108/MD-05-2017-0500. Recovered from https://www.scopus.com/record/display.uri?eid=2-s2.085061040909\&origin=resultslist\&sort=plf-

$\mathrm{f} \& \mathrm{src}=\mathrm{s} \& \mathrm{st} 1=$ municipal + tax + collection $\& \mathrm{st} 2=\& \mathrm{sid}=7 \mathrm{cbc} 9 \mathrm{bec} 885 \mathrm{c} 15 \mathrm{c} 2 \mathrm{f} 6487 \mathrm{ae} 5$ cae32eef $\&$ sot $=$ b\&sdt=b\&sl=39\&s=TITLE-ABS-

KEY\%28municipal+tax+collection\%29\&relpos=5\&citeCnt=2\&searchTerm $=$ 